\title{
The elemental abundances in the intracluster medium as observed with XMM-Newton
}

\author{
T. Tamura ${ }^{1}$, J. S. Kaastra ${ }^{1}$, J.W.A. den. Herder ${ }^{1}$, J. A. M. Bleeker ${ }^{1}$, and J. R. Peterson ${ }^{2}$ \\ 1 SRON National Institute for Space Research, Sorbonnelaan 2, 3584 CA Utrecht, The Netherlands \\ 2 KIPAC, Stanford University PO Box 20450 Stanford, CA 94309 \\ Received ?; accepted 2004-3-2
}

\begin{abstract}
XMM-Newton observations of 19 galaxy clusters are used to measure the elemental abundances and their spatial distributions in the intracluster medium. The sample mainly consists of X-ray bright and relaxed clusters with a $\mathrm{cD}$ galaxy. Along with detailed Si, S and Fe radial abundance distributions within 300-700 kpc in radius, the O abundances are accurately derived in the central region of the clusters. The Fe abundance maxima towards the cluster center, possibly due to the metals from the cD galaxy, are spatially resolved. The $\mathrm{Si}$ and $\mathrm{S}$ abundances also exhibit central increases in general, resulting in uniform Fe-Si-S ratios within the cluster. In contrast, the $\mathrm{O}$ abundances are in general uniform over the cluster. The mean $\mathrm{O}$ to Fe ratio within the cluster core is sub-solar, while that of the cluster scale is larger than the solar ratio. These measurements indicate that most of the Fe-Si-S and $\mathrm{O}$ in the intracluster medium have different origins, presumably in supernovae Ia and II, respectively. The obtained $\mathrm{Fe}$ and $\mathrm{O}$ mass are also used to discuss the past star formation history in clusters.
\end{abstract}

Key words. Galaxies: clusters: general - Galaxies: abundances - X-rays: galaxies: clusters

\section{Introduction}

Metals in the universe are being produced as a product of stellar evolution and ejected into intergalactic space via stellar winds and supernovae. The location of these metals can be measured by X-ray observations of the intra-cluster medium (ICM). These observations indicate that the ICM contains an amount of metals comparable to the total amount of metals found in galaxies (e.g. Tsuru 1991, Arnaud et al. 1992). Therefore, Xray measurement of the ICM metallicity distribution is a unique way to study the chemical history of galaxies. Most importantly, the total amount of metals and the abundance ratios among elements (e.g. $\mathrm{O} / \mathrm{Fe}$ ratio) constrain the total number of stars that have formed as well as their origin (i.e. the relative ratios of supernova types; Renzini et al.1993.

Measurements from ASCA and BeppoSAX observations revealed important properties of the ICM metallicity. These include an excess of $\mathrm{Fe}$ around $\mathrm{cD}$ galaxies (e.g. Fukazawa et al. 2000, De Grandi and Molendi 2001) and variations of the $\mathrm{Si} / \mathrm{Fe}$ ratio within a cluster (e.g. Finoguenov et al. 2000) and among clusters (e.g. Fukazawa et al. 1998). The variations in Si/Fe suggest that the metals in the ICM have at least two different origins, presumably SN Ia and SN II. The spatial resolution of these previous instruments was limited, causing the derived total amount of metals to depend on their assumed spatial distribution. The low spatial resolution combined with the limited

Send offprint requests to: T.Tamura

Correspondence to: T.Tamura@ plain.isas.ac.jp spectral resolution also introduced a systematic uncertainty in the temperature structure. This is particularly important in the central cool regions. This ambiguity in turn caused severe errors in the abundances of $\mathrm{Fe}$ and other elements. Furthermore, in most cases these measurements are limited to the $\mathrm{Fe}, \mathrm{Si}$ and S abundances.

Recent observations with Chandra and XMM-Newton have allowed a detailed study of the ICM metals, using an improved spatial resolution and sensitivity not only for the $\mathrm{Si}, \mathrm{S}$, and $\mathrm{Fe}$ lines but also for the $\mathrm{O}$ emission. For example, Tamura et al. (2001b) measured the spatial distribution of the $\mathrm{O}, \mathrm{Si}, \mathrm{S}$, and $\mathrm{Fe}$ in Abell 496 and found a difference between O and Si-SFe. David et al. (2001) used the Chandra data of the HydraA cluster to measure the $\mathrm{Si}$ and $\mathrm{Fe}$ abundances. In addition, the abundance distribution of several elements in the central region (70 kpc in radius) of the Virgo cluster was obtained with high accuracy (Finoguenov et al.2002, Gasteldello \& Molendi 2002, Matsushita et al.2003).

In this paper, we use the XMM-Newton EPIC and RGS instruments and measure the spatial distribution of heavy elements in 19 clusters. This systematic analysis enables us to compare the metal distribution in the sample. In addition, we may reduce the statistical errors on any given measurement when the variation in the sample is negligible. We selected the sample based on their X-ray flux and spatial size. Therefore the sample is limited to nearby X-ray bright clusters with a mean redshift of 0.06 . 
We obtained the radial distribution of $\mathrm{Fe}$ on a sub-arcmin scale for each cluster and confirmed the central increase of the $\mathrm{Fe}$ abundance within a typical radius of $100 \mathrm{kpc}$ in most cases. Furthermore, radial profiles of $\mathrm{O}, \mathrm{Si}$, and $\mathrm{S}$ relative to $\mathrm{Fe}$ have been measured in a fair number of clusters. We found that the $\mathrm{O}$ and $\mathrm{Si}-\mathrm{S}-\mathrm{Fe}$ abundances have different spatial distributions when we combine the results from several clusters.

Throughout this paper, we assume the Hubble constant to be $H_{0}=100 h \mathrm{~km} \mathrm{~s}^{-1} \mathrm{Mpc}^{-1}$ and use $90 \%$ confidence levels unless stated otherwise. Solar abundances are taken from Anders \& Grevesse (1989) with $\mathrm{Fe} / \mathrm{H}=4.68 \times 10^{-5}$.

\section{Observations}

We analyzed 19 clusters taken from GT and PV observations of XMM-Newton (Table 1 . The RGS exposure times are sometimes shorter than those reported in Peterson et al. (2003), because of a difference in the criteria for rejecting the exposure period with high background. We have previously reported detailed results for A 1795 (Tamura et al. 2001a), Sérsic 159-03 (Kaastra et al.2001), A 1835 (Peterson et al.2001), and A 496 (Tamura et al.2001b).

The present paper is one of a series of papers with a systematic study of cluster properties using similar data sets: soft $\mathrm{X}$-ray excess (Kaastra et al.2003a); central cooling region using RGS data (Peterson et al.2003); central cooling using EPIC (Kaastra et al. 2004). Here we focus on the spatial distribution of the metal abundances using both the EPIC and RGS data.

A detailed description of the XMM-Newton satellite and its instruments is found in Jansen et al. (2001), Turner et al. (EPIC/MOS; 2001), Strüder et al. (EPIC/pn; 2001), and den Herder et al. (RGS; 2001).

\section{The EPIC analysis}

\subsection{Spectral extraction}

In order to derive the spatial metallicity distribution, we used spatially-resolved EPIC spectra. We used the Science Analysis System (SAS; version 5.3.3) for the basic data processing. Then, we corrected the filtered events for the background, the telescope point spread function (PSF), and the effective area. We described the data correction and extraction extensively in Kaastra et al. (2004).

We removed high background time periods based on the number of counts in the $10-12 \mathrm{keV}$ band over the full field of view. Even after rejecting these high background events, there were still variations in the instrumental background from observation to observation. In order to correct for thiso weak variation, we sorted the cluster and blank sky data into subsets with the same $10-12 \mathrm{keV}$ count rate. Then, the frequency distributions of source count rate and a set of the blank sky spectra at different $10-12 \mathrm{keV}$ count rate level were used to determine the subtracted background spectrum. The source and background spectra were obtained from the same detector regions. To compensate for remaining variations in the instrumental background, we included a systematic uncertainty of at least $10 \%$ in the spectral fitting.
In both MOS and pn the $\mathrm{Al}$ lines around $1.48 \mathrm{keV}$, which are close to the line positions of H-like and He-like Mg ions, are strong. Therefore, we did not attempt to derive the $\mathrm{Mg}$ abundance except for bright cluster central regions. In addition, in the MOS, there are instrumental Si lines around $1.74 \mathrm{keV}$. Compared to the $\mathrm{Al}$ lines, these lines are relatively weak and not as close to that from astrophysical Si ions. Therefore, an additional uncertainty in the Si background lines produces no change in our result. We verified this using representative cluster data.

In the low energy band, the cosmic background dominates the instrumental background and varies spatially by more than $10 \%$. Therefore, we included larger systematic errors in the soft $\mathrm{X}$-ray band as shown in Table 2

In all observations the bright X-ray point sources were removed. Typically, a median of 8 point sources (for MOS1 and MOS2) or 15 (for pn) were discarded.

Then, we made a background subtracted radial profile for each energy. This profile was corrected for the exposure, telescope PSF, and vignetting. We assumed energy-independent PSF correction (Kaastra et al. 2004). Therefore it affects only the spectral normalization, not its shape. On the other hand, the correction for the vignetting due to the telescope is dependent on the X-ray position and energy. The MOS cameras also have a correction due to obscuration of the Reflection Grating Array that is dependent on position and energy as well.

From the corrected radial profile for each energy band, we extracted projected spectra from concentric annuli centered on the X-ray maximum with outer radii of $0.5,1,2,3,4,6,9,12$, 16 arcminutes. In addition, assuming spherical symmetry, we derived deprojected spectra from shells with the same radial binning as above. The deprojected spectrum represents a set of count rates in a spherical shell between the two spheres and can be given by a linear combination of the observed number of counts in the (projected) surface brightness profile. Note that we ignored X-ray emission outside the field of view and that the spectra of the outermost annulus $\left(12^{\prime}-16^{\prime}\right)$ are used only for the deprojection for the inner shells. We used different innermost and outermost bins among our clusters as shown in Table 1 depending upon the statistical quality of the spectra.

We do not completely take into account the spectral mixing due to the PSF. Therefore the true profiles of temperature and metallicity could be much steeper than the present results. This effect should be small since the the radial bin size is larger than the PSF width (a half energy width of $15^{\prime \prime}$ ).

\subsection{Spectral fitting}

In most cases, the instrumental background dominates the source emission above $\sim 8 \mathrm{keV}$. Between $7-9 \mathrm{keV}$, the pn instrumental background contains strong spectral lines. Furthermore, when we fitted the pn spectra of bright clusters with representative models, we found systematic residuals below the Fe-L lines. This is probably due to uncertainties in the energy redistribution response at the low energy. Taking this into account, we restricted our analysis for the MOS and pn spectra between 0.3 and $8 \mathrm{keV}$ and between 0.7 and 
Table 1. Cluster sample.

\begin{tabular}{|c|c|c|c|c|c|c|c|c|c|c|}
\hline \multirow[t]{2}{*}{ Cluster } & \multirow[t]{2}{*}{ redshift $^{a}$} & \multirow{2}{*}{$\begin{array}{l}k T^{\mathrm{b}} \\
(\mathrm{keV})\end{array}$} & \multirow{2}{*}{$\begin{array}{c}n_{\mathrm{H}}{ }^{\mathrm{c}} \\
\left(10^{24} \mathrm{~m}^{-2}\right)\end{array}$} & \multirow[t]{2}{*}{$\mathrm{C}^{\mathrm{d}}$} & \multirow{2}{*}{$\begin{array}{c}\text { XMM } \\
\text { revolution }\end{array}$} & \multicolumn{3}{|c|}{ Exposure time $(\mathrm{ks})^{\mathrm{e}}$} & \multicolumn{2}{|c|}{$\overline{R^{f}}$} \\
\hline & & & & & & MOS & $\mathrm{pn}$ & RGS & IN & OUT \\
\hline NGC 533 & 0.0175 & 1.3 & 3.00 & $\bar{C}$ & 195 & 38 & 31 & 49 & 0 & 6 \\
\hline A 262 & 0.0155 & 2.2 & $8.94(5.33)$ & $\mathrm{C}$ & 203 & 24 & 15 & 35 & 0 & 12 \\
\hline Sérsic $159-03$ & 0.0572 & 2.4 & 1.79 & $\mathrm{C} / \mathrm{SE}$ & 77 & 31 & 28 & 36 & 0 & 9 \\
\hline MKW 9 & 0.0402 & 2.6 & 4.18 & C & 311 & 22 & 13 & - & 0 & 3 \\
\hline 2A $0335+096$ & 0.0344 & 3.0 & $28.71(18.64)$ & M & 215 & 11 & 5 & 18 & 0 & 9 \\
\hline A 2052 & 0.0356 & 3.1 & 2.91 & $\mathrm{M} / \mathrm{SE}$ & 128 & 30 & 23 & 28 & 0 & 12 \\
\hline Hydra A (A 780) & 0.0550 & 3.4 & 4.80 & M & 183 & 17 & 3 & 25 & 0 & 6 \\
\hline MKW 3s & 0.0455 & 3.5 & 2.89 & $\mathrm{M} / \mathrm{SE}$ & 129 & 37 & 32 & - & 0 & 12 \\
\hline A 4059 & 0.0466 & 4.0 & 1.06 & M & 176 & 34 & 6 & 44 & 0 & 9 \\
\hline A 1837 & 0.0707 & 4.4 & 4.38 & M & 200 & 48 & 40 & - & 0 & 6 \\
\hline A 496 & 0.0322 & 4.4 & $6.44(4.23)$ & M & 211 & 9 & 5 & 18 & 0 & 12 \\
\hline A 3112 & 0.0756 & 4.5 & 2.61 & $\mathrm{M} / \mathrm{SE}$ & 191 & 22 & 16 & 24 & 0 & 9 \\
\hline A 1795 & 0.0639 & 5.8 & 1.01 & $\mathrm{M} / \mathrm{SE}$ & 100 & 29 & 22 & 37 & 0 & 9 \\
\hline A 399 & 0.0706 & 6.2 & 10.90 & $\mathrm{H}$ & 127 & 13 & 6 & - & 0.5 & 9 \\
\hline Perseus (A 426) & 0.0179 & 6.5 & 14.90 & $\mathrm{H}$ & 210 & 43 & 33 & - & 0 & 12 \\
\hline A 1835 & 0.2541 & 7.2 & 2.32 & $\mathrm{H}$ & 101 & 0 & 25 & 29 & 0 & 6 \\
\hline Coma (A 1656) & 0.0240 & 7.5 & 0.89 & $\mathrm{H} / \mathrm{SE}$ & 86 & 15 & 13 & - & 0.5 & 12 \\
\hline A 754 & 0.0561 & 8.0 & $5.67(4.37)$ & $\mathrm{H}$ & 262 & 14 & 11 & - & 0.5 & 12 \\
\hline A 3266 & 0.0614 & 8.7 & 1.60 & $\mathrm{H}$ & 153 & 24 & 19 & - & 0.5 & 12 \\
\hline Virgo $^{g}$ & 0.0027 & 2.6 & 1.80 & $\mathrm{C}$ & 97 & 34 & 25 & - & 0 & 12 \\
\hline
\end{tabular}

a Redshift, to be used for the distance estimate.

b The temperature outside the central cool region obtained from our EPIC analysis (Kaastra et al. 2004).

c Galactic column density to be used for the spectral modeling. In most cases, these are obtained from H I map (Dickey \& Lockman 1990 ). In other cases, we present the used (X-ray) value with the $\mathrm{H}$ I value in parentheses.

d Classification of clusters used in the present paper. "C", "M", and "H" indicate cool, medium-temperature, and hot clusters, respectively. "SE" indicates clusters which show soft X-ray excess.

e Net exposure time, after rejection of high background time periods. We did not use the RGS data for clusters indicated by "-", because of poor statistics.

f Innermost and outermost radii for the EPIC spectral analysis in arcmin.

$g$ The data for the Virgo cluster are used only for the EPIC calibration.

$7.0 \mathrm{keV}$ energy ranges, respectively. The three spectra (MOS1, MOS2, and pn) were fitted simultaneously with the same spectral model, but we allowed the relative normalizations to be free parameters.

Table 2. Systematic errors relative to the flux. These were used for the EPIC spectral modeling and error estimates.

\begin{tabular}{ccccc}
\hline Energy band $(\mathrm{keV})$ & $0.3-0.5$ & $0.5-0.7$ & $0.7-2.0$ & $2.0-8.0$ \\
\hline source & 5 & 5 & 5 & 10 \\
background & 35 & 25 & 15 & 10 \\
\hline
\end{tabular}

We used a collisional ionization equilibrium model (CIE; implemented in the SPEX package ${ }^{1}$; Mewe et al. 1985, Kaastra 1992, Liedahl et al. 1995) modified by photoelectric absorption. The abundance ratios of $\mathrm{C}$ and $\mathrm{N}$ were fixed to the solar values, based on the best-fit value derived from the RGS in the central region of M87 (Sakelliou et al.2002). Those of O, Ne, $\mathrm{Mg}, \mathrm{Si}, \mathrm{S}, \mathrm{Ar}, \mathrm{Ca}$, and $\mathrm{Fe}$ were left free, while those of other heavy elements were fixed to that of $\mathrm{Fe}$ (e.g., $\mathrm{Ni} / \mathrm{Fe}=1$ ). Thus

\footnotetext{
1 see http://www.sron.nl/divisions/hea/spex/
}

the free parameters are the normalization, temperature $(k T)$, and metal abundances (O, Ne, Mg, Si, S, Ar, Ca, and Fe).

We used a fixed column density for each cluster as shown in Table 1 These column densities have been adjusted to match the observed EPIC spectra as follows. In most cases, the bestfit value is consistent with the Galactic one obtained from the H I map (Dickey \& Lockman 1990). In those cases we kept the column density fixed to the Galactic value. In A 262, 2A 0335+096, A 496 and A 754, however, the data require additional absorption, possibly caused by Galactic gas and dust (we investigated these individual cases in more detail in Kaastra et al.2003a).

Usually, the best-fit parameters (normalization, $k T$, and abundances) are derived at the same time by fitting the spectrum in a wide energy band. If however the response matrix is not accurate due to a calibration error in some wide energy band of the spectrum, the modeled local continuum may differ slightly from the actually observed continuum in that wide energy band. This could result in errors on the derived equivalent width of lines in that band and hence in errors on the obtained abundances. In fact, when we used this global fitting method with the MOS and pn spectra separately with bright 
clusters, we found that the MOS gives systematically higher $\mathrm{Si}$ and $\mathrm{S}$ abundances by $\sim 0.2$ of the solar value than the pn does. To avoid such errors as much as possible, we derive the parameters in two steps. First, we measure the best-fit $k T$ by fitting the wide energy band spectra. Secondly, we fix $k T$, use the local spectra in the limited energy band close to the relevant elemental-ion lines and derive the abundance by allowing the normalization to be free. We used energy bands of 0.35 1.0 (for O), 0.33-2.2 ( $\mathrm{Ne}$ and $\mathrm{Mg}$ ), 1.2-3.2 ( $\mathrm{Si}$ and $\mathrm{S}$ ), 2.7-4.7 (Ar and $\mathrm{Ca}$ ), all in keV. An exception is the Fe abundance, for which we use the wide energy band to include both the Fe-L and Fe-K lines simultaneously.

We analyzed both projected and deprojected spectra. The projected spectra in general have better statistics but poorer fits for each radius, mainly because of the contamination from different radii with different $k T$. Also, the derived spatial distribution of the temperature changes by using deprojection, in particular in the inner regions (See Fig.2 in Tamura et al. 2001b for an example). To obtain an accurate abundance distribution, proper modeling of the temperature distribution is necessary. Therefore, we present the results of the deprojected spectra.

\subsection{Systematic uncertainties}

We used several calibration sources to estimate the uncertainty in the response calibration. Based on this, we place conservatively systematic errors on the source spectra. Table 2 summarizes the values of these systematic errors that we used. These errors along with those of the background were added in quadrature in the binned spectra.

To assess additional systematic errors, we derived abundances ( $\mathrm{Si}, \mathrm{S}$, and $\mathrm{Fe}$ ) separately from the MOS and pn spectra in representative clusters (M 87, A 262, and A 1795). For the $\mathrm{Fe}$ abundance determination, we separately used $\mathrm{Fe}-\mathrm{L}$ and $\mathrm{Fe}-$ $\mathrm{K}$ lines. The results are shown in Fig. 10 The pn detector still gave systematically smaller $\mathrm{Si}$ and $\mathrm{Fe}$ abundances than MOS. The differences were less than $10-20 \%$ of the obtained values and less than the typical statistical errors for each spectrum. In other cases, the two instruments provided consistent results. Because the O VIII Ly $\alpha$ line was contained only in the MOS spectrum in our case, we cannot check the $\mathrm{O}$ abundance in a similar way. Nevertheless, we confirmed that the $\mathrm{O}$ abundances obtained from MOS are consistent with those obtained from RGS as shown below (Fig. 22. The three types of $X M M$-Newton instruments have different energy responses and have been calibrated independently, except for the telescope response. Therefore the above consistency checks indicate that there is negligible additional systematic error above the values that we already assigned compared to the statistical uncertainty in our sample.

\subsection{Results}

In general, the single temperature (1T) model provided a good fit to the spectra. Figures 3 and 4 show examples of the fit. However, the spectra from the following regions cannot be described by the $1 \mathrm{~T}$ model $\left(\chi^{2} / \nu>1.2\right)$. NGC $533\left(0^{\prime}-1^{\prime} .0\right)$,
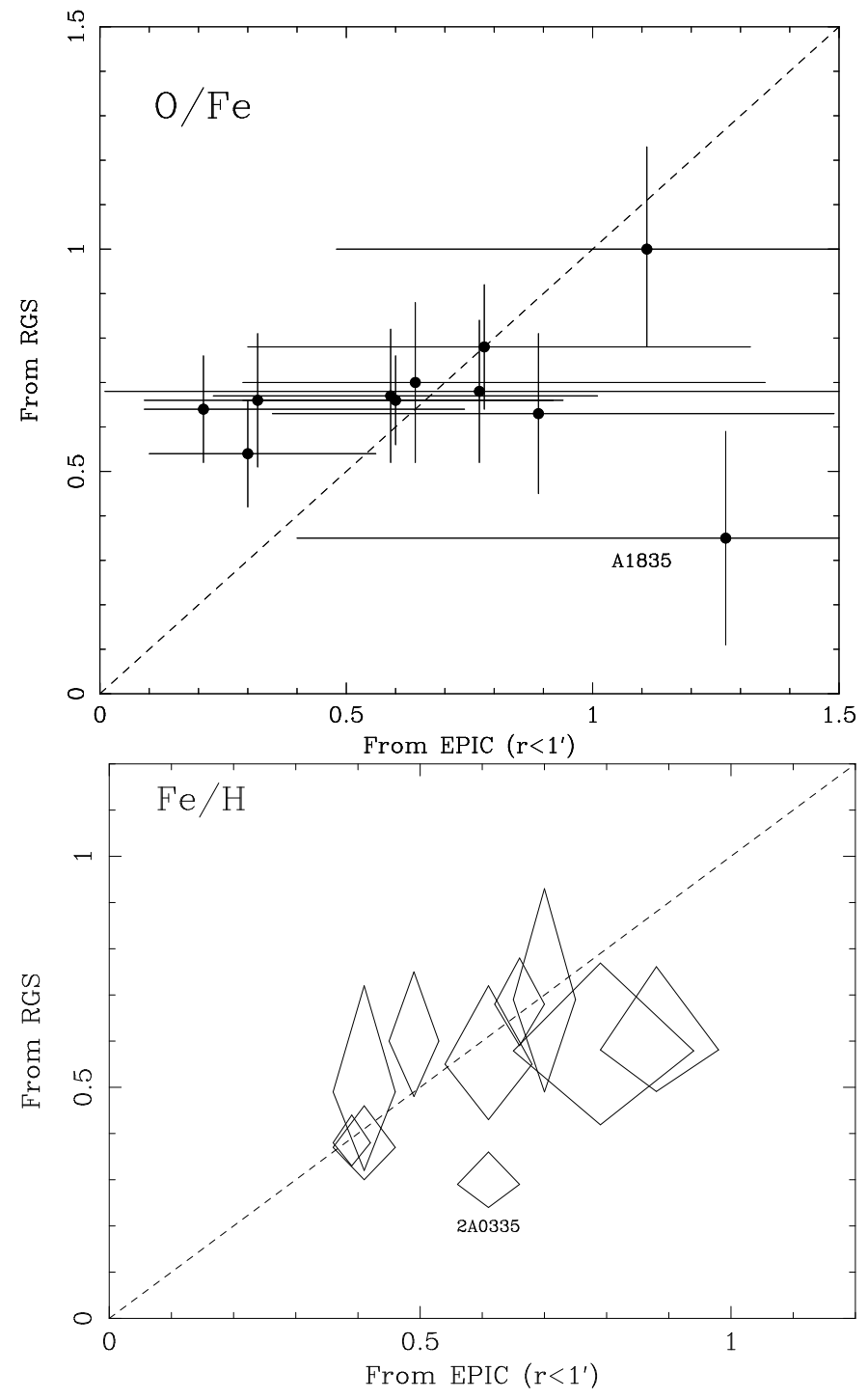

Fig. 2. Comparison between the EPIC and RGS results for $\mathrm{O} / \mathrm{Fe}$ and $\mathrm{Fe} / \mathrm{H}$.

A $262\left(0^{\prime}-0^{\prime} .5\right), 2 \mathrm{~A} 0335+096\left(0^{\prime}-1^{\prime} .0\right)$, MKW $3 \mathrm{~s}\left(6^{\prime}-12^{\prime}\right)$, A $2052\left(0^{\prime}-0^{\prime} .5,9^{\prime}-12^{\prime}\right)$, A $4059\left(0^{\prime}-0^{\prime} .5\right)$, A 3112 $\left(1^{\prime}-2^{\prime}, 3^{\prime}-6^{\prime}\right)$, and Perseus $\left(0^{\prime}-2^{\prime}, 3^{\prime}-4^{\prime}\right)$.

In the inner regions of some clusters $\left(r<1^{\prime}\right)$, the data exhibit a clear deviation from the 1T model. A major part of this discrepancy can be removed by adding an additional cool component. In addition, some spectra in the outer regions cannot be modeled by the $1 \mathrm{~T}$ model, probably because of soft excess emission below $0.5 \mathrm{keV}$. We reported earlier a detailed analysis of this soft excess in Sérsic 159-03, A 2052, A 1795, and Coma (Kaastra et al. 2003a). In addition to these clusters, A 3112 also shows a similar excess above the $1 \mathrm{~T}$ model (Nevalainen et al.2003, Kaastra et al.2003c).

To describe the multi-temperature structure in the central regions, we used a two temperature model (2T). In Kaastra et al. (2004) and Peterson et al. (2003) we found that in almost all cases, at each radius there is negligible emission from gas with a temperature less than one-third to half of the ambient temper- 

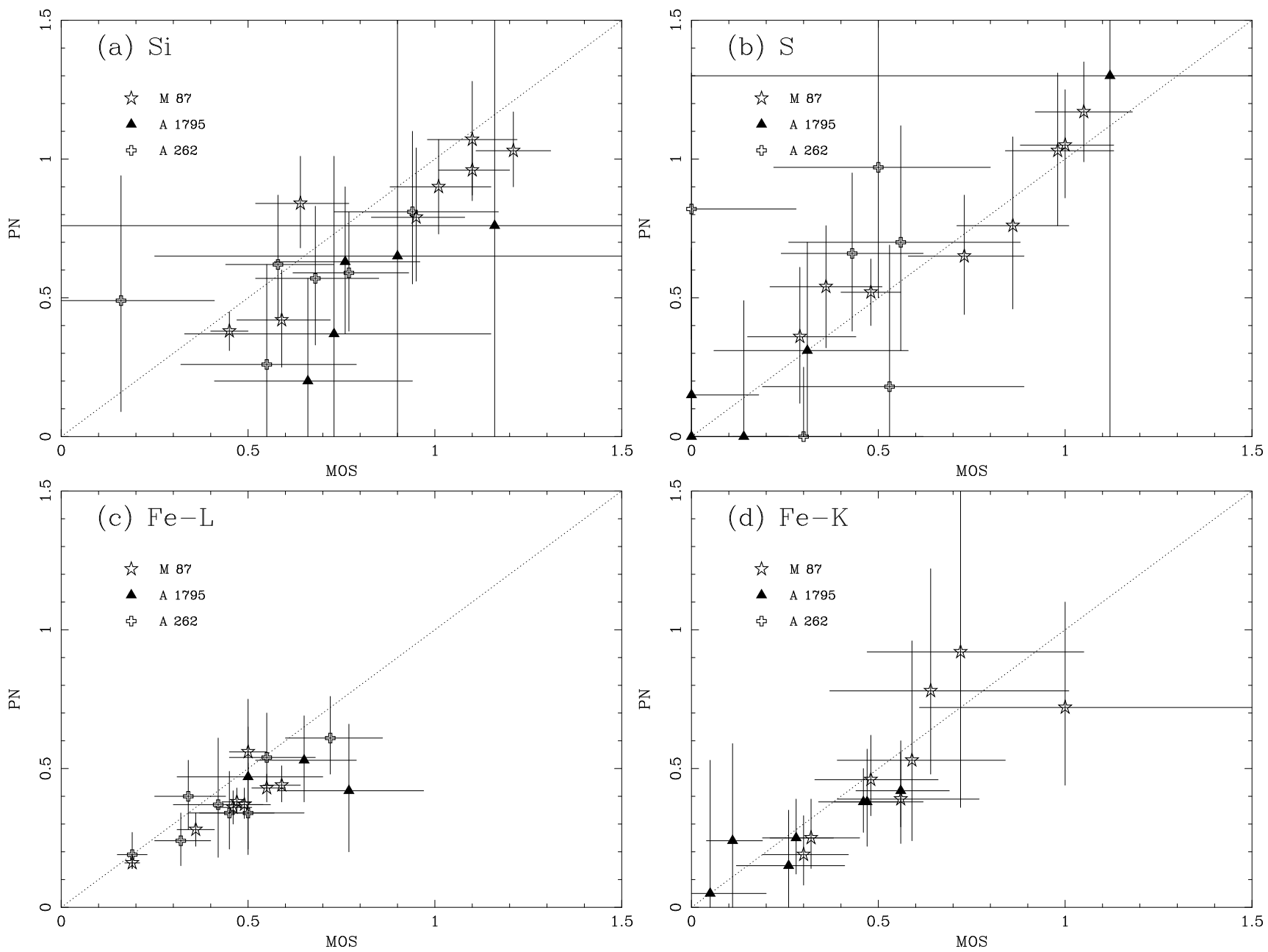

Fig. 1. Comparison between the MOS and pn results for the $\mathrm{Si}(\mathrm{a}), \mathrm{S}(\mathrm{b})$, and $\mathrm{Fe}$ (c and d) abundances. We used spectra from several regions of M 87 (the brightest cluster at low energies), A 262 (temperature $2 \mathrm{keV}$ ), and A 1795 (temperature 4-6 keV) as representative samples.

ature. Therefore, we fixed the temperature separation between both components as $T_{0}=2 T_{1}$, where $T_{0}$ and $T_{1}$ are the two temperatures.

For the inner regions of A 262, 2A 0335+096, A 2052 and A 4059, the $2 \mathrm{~T}$ model improved the fit (Table 3). In some cases, the $\mathrm{Fe}$ abundance changed significantly as compared to the single temperature fit (the so-called Fe-bias, see for example Buote 2000 and Molendi \& Gastaldello 2001). For these clusters, we use the abundances derived from this $2 \mathrm{~T}$ model for the discussion below. For the inner regions in some other clusters (e.g. A 496 and A 1795), the $2 \mathrm{~T}$ model sometimes provides a slightly better fit over the 1T model. However, in these cases the changes in the obtained $\mathrm{Fe}$ abundances are small compared to the statistical errors. This is because the deviation from isothermality within each radius is relatively small.

In some cases, the fit is improved further by de-coupling $T_{0}$ with $T_{1}$. However, even in those cases, the change in the Fe abundance from the above $2 \mathrm{~T}$ model is small ( 10\%) compared to the errors.

In all cases except for A 3226, Coma and A 754, the temperature decreases towards the cluster center (Kaastra et al.
2004). In Fig. 5 we present the abundances as derived from the acceptable fits $\left(\chi^{2} / \nu<1.3\right)$ of either the $1 \mathrm{~T}$ or $2 \mathrm{~T}$ models. In Tables 44, we show average values over three different spatial scales. We also present average values for cool $\left(k T_{\mathrm{ICM}}<3 \mathrm{keV}\right)$, medium-temperature, and hot clusters $\left(k T_{\mathrm{ICM}}>6 \mathrm{keV}\right)$ in Table 6 Furthermore, we separately show the results for some clusters which exhibit soft excess ("SE" in Table 11. Fig. 6 shows abundances averaged over (50200) $h^{-1} \mathrm{kpc}$ for each cluster as a function of the ICM temperature. Below we summarize the main results of the Fe abundance, $\mathrm{O} / \mathrm{Fe}, \mathrm{Si} / \mathrm{Fe}$, and $\mathrm{S} / \mathrm{Fe}$ ratios.

In all cool and medium-temperature clusters except MKW 9, the faintest one, we detected a central increase in the $\mathrm{Fe}$ abundance; the abundance starts to increase at (50100) $h^{-1} \mathrm{kpc}$ in radius from $0.2-0.4$ solar to $0.6-0.8$ solar towards the center. Except for the Perseus cluster, we found no significant $\mathrm{Fe}$ increase in the inner regions of the hot clusters (See Table 5]. However, the best-fit value for each cluster suggests the Fe increase in all cases and the average Fe value over the hot clusters also indicates a central enhancement (Table 6 ) 
Table 3. Spectral fit results for the inner regions of some clusters for a single temperature (1T fit) and a two temperature (2T fit) model. We present no error when the fit is largely unacceptable.

\begin{tabular}{lccccccc}
\hline cluster & $\mathrm{R}^{\mathrm{a}}$ & $\begin{array}{c}k T^{\mathrm{b}} \\
(\mathrm{keV})\end{array}$ & $\begin{array}{c}1 \mathrm{~T} \mathrm{fit} \\
\mathrm{Fe} / \mathrm{H} \\
(\text { solar })\end{array}$ & $\chi^{2} / \nu$ & $\begin{array}{c}k T_{0}^{\mathrm{b}} \\
(\mathrm{keV})\end{array}$ & $\begin{array}{c}\text { Fe/H fit } \\
(\text { solar })\end{array}$ & $\chi^{2} / \nu$ \\
\hline A 262 & $0.0-0.5$ & 1.0 & 0.31 & $457 / 227$ & $1.5 \pm 0.07$ & $0.85 \pm 0.1$ & $274 / 226$ \\
& $0.5-1.0$ & $1.4 \pm 0.04$ & $0.58 \pm 0.07$ & $242 / 227$ & $1.6 \pm 0.1$ & $0.89 \pm 0.1$ & $214 / 226$ \\
2A 0335+096 & $0.0-0.5$ & 1.4 & 0.32 & $400 / 228$ & $1.7 \pm 0.1$ & $0.55 \pm 0.07$ & $298 / 227$ \\
& $0.5-1.0$ & $1.8 \pm 0.05$ & $0.5 \pm 0.05$ & $275 / 227$ & $2.1 \pm 0.1$ & $0.64 \pm 0.06$ & $210 / 227$ \\
A 2052 & $0.0-0.5$ & 1.4 & 0.35 & $574 / 228$ & $2.0 \pm 0.1$ & $0.62 \pm 0.05$ & $312 / 227$ \\
& $0.5-1.0$ & $2.6 \pm 0.06$ & $0.71 \pm 0.06$ & $255 / 228$ & $2.9 \pm 0.2$ & $0.67 \pm 0.06$ & $213 / 227$ \\
A 4059 & $0.0-0.5$ & 2.2 & 0.63 & $283 / 226$ & $2.6 \pm 0.4$ & $0.60 \pm 0.2$ & $258 / 223$ \\
\hline
\end{tabular}

a Radial range (arcmin).

b The best-fit and hotter temperature for the $1 \mathrm{~T}$ fit and for the $2 \mathrm{~T}$ fit, respectively.

Table 6. Average abundances obtained from the EPIC analysis for the cool, medium-temperature, hot, and soft excess clusters. Same notation as Table 4

\begin{tabular}{|c|c|c|c|c|c|c|c|c|}
\hline $\bar{R}$ & $\mathrm{Fe} / \mathrm{H}$ & $\mathrm{O} / \mathrm{Fe}$ & $\mathrm{Ne} / \mathrm{Fe}$ & $\mathrm{Mg} / \mathrm{Fe}$ & $\mathrm{Si} / \mathrm{Fe}$ & $\mathrm{S} / \mathrm{Fe}$ & $\mathrm{Ar} / \mathrm{Fe}$ & $\mathrm{Ca} / \mathrm{Fe}$ \\
\hline \multicolumn{9}{|c|}{$\operatorname{Cool}(k T<3 \mathrm{keV})$} \\
\hline \#0 & $0.57 \pm 0.04$ & $0.4 \pm 0.2$ & $1.4 \pm 0.4$ & $0.6 \pm 0.3$ & $1.6 \pm 0.2$ & $1.4 \pm 0.3$ & $1.9 \pm 1.5$ & $3.1 \pm 2.1$ \\
\hline$\# 1$ & $0.42 \pm 0.08$ & $1.9 \pm 0.9$ & $1.0 \pm 0.8$ & - & $1.2 \pm 0.5$ & $0.7 \pm 0.6$ & - & - \\
\hline \multicolumn{9}{|c|}{ medium-T $(3<k T<6 \mathrm{keV})$} \\
\hline$\# 0$ & $0.60 \pm 0.04$ & $0.7 \pm 0.4$ & $1.8 \pm 0.5$ & $0.3 \pm 0.3$ & $1.6 \pm 0.3$ & $1.0 \pm 0.3$ & $1.1 \pm 0.7$ & $2.9 \pm 1.3$ \\
\hline$\# 1$ & $0.39 \pm 0.03$ & $1.2 \pm 0.5$ & $1.3 \pm 0.5$ & - & $1.4 \pm 0.4$ & $0.5 \pm 0.3$ & $1.2 \pm 1.0$ & $3.3 \pm 1.6$ \\
\hline$\# 2$ & $0.32 \pm 0.08$ & $4.0 \pm 2.5$ & $1.5 \pm 1.2$ & - & $1.3 \pm 0.9$ & $1.7 \pm 1.1$ & $1.3 \pm 2.8$ & - \\
\hline \multicolumn{9}{|c|}{ Hot $(k T>6 \mathrm{keV})$} \\
\hline$\# 0$ & $0.48 \pm 0.12$ & $1.2 \pm 1.2$ & $2.6 \pm 1.9$ & $1.3 \pm 2.2$ & $2.2 \pm 1.9$ & $0.5 \pm 1.6$ & - & - \\
\hline \#1 & $0.34 \pm 0.05$ & $2.1 \pm 1.7$ & $3.6 \pm 2.0$ & - & $1.9 \pm 1.7$ & $1.8 \pm 1.8$ & $3.3 \pm 4.1$ & - \\
\hline$\# 2$ & $0.25 \pm 0.05$ & - & $3.3 \pm 3.4$ & - & - & $3.1 \pm 4.0$ & - & - \\
\hline \multicolumn{9}{|c|}{ Soft excess clusters } \\
\hline$\# 0$ & $0.57 \pm 0.02$ & $0.6 \pm 0.2$ & $1.6 \pm 0.3$ & $0.4 \pm 0.2$ & $1.4 \pm 0.2$ & $0.7 \pm 0.2$ & $0.8 \pm 0.4$ & $2.4 \pm 0.7$ \\
\hline$\# 1$ & $0.32 \pm 0.02$ & $0.8 \pm 0.3$ & $0.8 \pm 0.4$ & - & $1.5 \pm 0.3$ & $0.4 \pm 0.3$ & $1.1 \pm 0.8$ & $3.1 \pm 1.3$ \\
\hline$\# 2$ & $0.17 \pm 0.04$ & $1.5 \pm 1.2$ & $2.6 \pm 1.2$ & - & $2.2 \pm 1.0$ & $1.8 \pm 1.1$ & $1.1 \pm 3.3$ & - \\
\hline
\end{tabular}

In the outer regions, (200-500) $h^{-1} \mathrm{kpc}$ in radius for all clusters, the Fe abundance is $0.2-0.3$, without a significant dependence on the ICM temperature (Fig. 6). Exceptions are the soft excess clusters, which show relatively smaller Fe abundances down to 0.1 solar. These low Fe abundances may not be the actual values due to a spectral contamination by the soft excess emission, which was not taken into account in the present analysis. Further careful analysis is necessary to take into account the soft excess emission and derive the accurate Fe abundance. This is beyond the scope of the present paper.

The error on the Si abundance determination is larger than on iron. Nevertheless, there is also an indication of a central increase in the $\mathrm{Si}$ abundance. In fact, the $\mathrm{Si} / \mathrm{Fe}$ ratio is radially uniform at an average value of 1-2 solar (Fig. 56). Within the error bars, there is no radial change of the $\mathrm{Si} / \mathrm{Fe}$ ratio for each cluster and among different clusters (Fig. 6). The radial distribution of the $\mathrm{S}$ abundance is similar to that of $\mathrm{Si}$; the $\mathrm{S} / \mathrm{Si}$ ratios are consistent with the solar ratio.

In the cool and medium-temperature clusters, the measured $\mathrm{O} / \mathrm{Fe}$ abundance ratio at the cluster center is $0.4-0.7$ relative to the solar value. This is consistent with the values obtained from the RGS (as shown later). For the hot clusters, the errors on the $\mathrm{O}$ abundance are too large to allow a measurement of spatial variations. Contrary to $\mathrm{Fe}$ and $\mathrm{Si}$, the $\mathrm{O}$ abundance shows no significant central increase. The $\mathrm{O} / \mathrm{Fe}$ ratio averaged over the cool and medium-temperature clusters increases towards large radii to a value of a few times solar.

$\mathrm{The} \mathrm{Ne}, \mathrm{Mg}, \mathrm{Ar}$, and $\mathrm{Ca}$ abundances have larger errors. We only show the average abundance of these elements in Table 6 In general, these abundances relative to $\mathrm{Fe}$ are consistent with the solar ratio.

\section{The RGS analysis}

\subsection{Analysis method}

The RGS events were filtered using both the dispersion vs. cross-dispersion and dispersion vs. pulse height windows. We used a spectral extraction region of $1^{\prime} .0$ in full-width in the cross-dispersion direction centered on the X-ray maximum. This corresponds to a physical size of $50 h^{-1} \mathrm{kpc}$ for a mean 


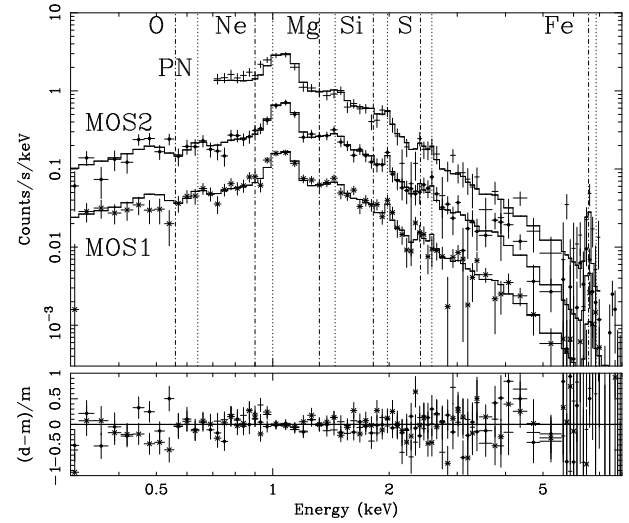

Fig. 3. An example of the spectral fitting. The spectra of A 262 between $1^{\prime}-2^{\prime}$ in deprojected radius are shown. The MOS1 and pn spectra are multiplied by factors of 0.25 and 2, respectively, for clarity. The data are shown with the model as a histogram. Expected line positions for $\mathrm{H}$ - and $\mathrm{He}$-like ions are indicated by vertical lines. The bottom panel shows the fit residuals [(data-model)/model]. In this case, we obtain a temperature of $1.9 \mathrm{keV}$ and abundances for $\mathrm{O}, \mathrm{Si}, \mathrm{S}$, and $\mathrm{Fe}$ of $0.5,1.0,1.0$, and 0.7 times solar, respectively.

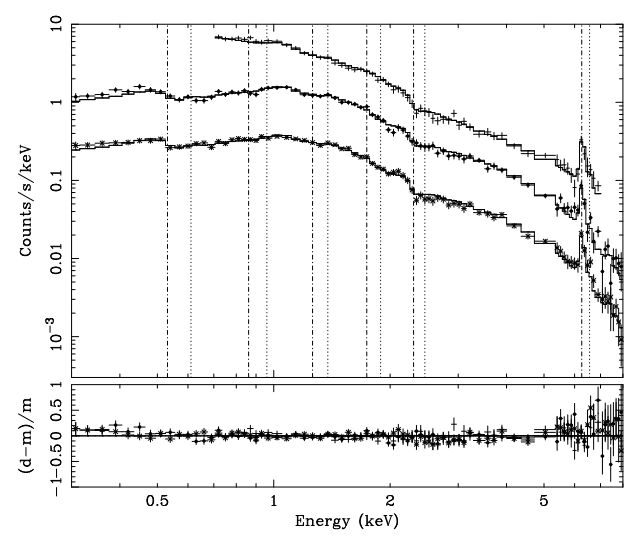

Fig. 4. Same as the previous figure, but for the spectra of A 1795 between $1^{\prime}-2^{\prime}$ in deprojected radius. We obtain a temperature of $5.3 \mathrm{keV}$ and abundances for $\mathrm{O}, \mathrm{Si}, \mathrm{S}$, and $\mathrm{Fe}$ of 0.20 , $0.7,0.0$, and 0.4 times solar, respectively.

redshift of 0.06 . Generally, most of the source photons in the RGS aperture are inside this extraction region. We used a pulse height width selection that included $90 \%$ of all photons for a point source.

Our targets have a larger spatial extent than the instrumental spatial resolution, resulting in a broadening of the line spread function (LSF). Here we modeled the source brightness using the observed MOS image for each target. Then, we corrected for this effect by convolving the cluster brightness profile with the RGS response for a point source. Because the dependence of the LSF on the off-axis angle within $\pm 3^{\prime}$ is smaller than 10 $30 \%$ and the cluster emission is dominated by emission within that spatial range, we ignore this dependence of the LSF.

We have estimated the background spectrum and its variability using several blank-sky field observations $(\sim 400 \mathrm{ks}$ in total exposure; Tamura et al.2003b). The count rate variations
Table 4. Abundances (scaled to the solar ratio) obtained from the EPIC analysis for each cluster. Values with errors larger than 3 times solar are not presented (indicated by - ).

\begin{tabular}{|c|c|c|c|c|}
\hline $\mathrm{R}^{\mathrm{a}}$ & $\mathrm{Fe} / \mathrm{H}$ & $\overline{\mathrm{O} / \mathrm{Fe}}$ & $\mathrm{Si} / \mathrm{Fe}$ & $\mathrm{S} / \mathrm{Fe}$ \\
\hline \multicolumn{5}{|c|}{ NGC 533} \\
\hline$\# 0$ & $0.39 \pm 0.06$ & $0.3 \pm 0.7$ & $1.6 \pm 0.7$ & $2.8 \pm 1.3$ \\
\hline \#1 & $0.26 \pm 0.09$ & $1.9 \pm 1.9$ & $1.7 \pm 1.2$ & $1.1 \pm 1.7$ \\
\hline \multicolumn{5}{|c|}{ A 262} \\
\hline$\# 0$ & $0.67 \pm 0.05$ & $0.6 \pm 0.3$ & $1.4 \pm 0.2$ & $1.0 \pm 0.3$ \\
\hline$\# 1$ & $0.34 \pm 0.04$ & $1.8 \pm 0.9$ & $1.2 \pm 0.4$ & $0.7 \pm 0.5$ \\
\hline \multicolumn{5}{|c|}{ A 1837} \\
\hline$\# 0$ & $0.64 \pm 0.14$ & $0.5 \pm 1.6$ & $2.8 \pm 1.6$ & $1.0 \pm 1.4$ \\
\hline \#1 & $0.39 \pm 0.07$ & $0.9 \pm 1.1$ & $0.9 \pm 0.7$ & $0.4 \pm 0.8$ \\
\hline$\# 2$ & $0.27 \pm 0.13$ & $1.7 \pm 3.0$ & $1.0 \pm 1.6$ & $3.7 \pm 2.9$ \\
\hline \multicolumn{5}{|c|}{ Sérsic $159-3$} \\
\hline$\# 0$ & $0.39 \pm 0.03$ & $0.6 \pm 0.4$ & $1.3 \pm 0.2$ & $0.8 \pm 0.3$ \\
\hline$\# 1$ & $0.23 \pm 0.03$ & $0.8 \pm 0.8$ & $1.4 \pm 0.6$ & $0.5 \pm 0.5$ \\
\hline \#2 & $0.09 \pm 0.05$ & - & $1.5 \pm 1.7$ & $1.5 \pm 2.7$ \\
\hline \multicolumn{5}{|c|}{ MKW 9} \\
\hline$\# 0$ & $0.63 \pm 0.15$ & $0.1 \pm 0.5$ & $1.9 \pm 0.7$ & $1.4 \pm 0.8$ \\
\hline$\# 1$ & $0.97 \pm 0.45$ & - & $0.2 \pm 0.8$ & $0.0 \pm 0.7$ \\
\hline \multicolumn{5}{|c|}{ 2A $0335+096$} \\
\hline$\# 0$ & $0.60 \pm 0.04$ & $1.6 \pm 0.7$ & $1.0 \pm 0.2$ & $0.8 \pm 0.3$ \\
\hline \#1 & $0.42 \pm 0.07$ & $1.8 \pm 1.7$ & $1.2 \pm 0.6$ & $0.0 \pm 0.4$ \\
\hline \#2 & $0.31 \pm 0.17$ & - & $0.3 \pm 1.1$ & $1.5 \pm 1.9$ \\
\hline \multicolumn{5}{|c|}{ MKW 3s } \\
\hline$\# 0$ & $0.68 \pm 0.06$ & $0.5 \pm 0.4$ & $1.4 \pm 0.3$ & $0.9 \pm 0.4$ \\
\hline \#1 & $0.31 \pm 0.03$ & $1.3 \pm 0.9$ & $1.5 \pm 0.6$ & $0.5 \pm 0.5$ \\
\hline$\# 2$ & $0.09 \pm 0.06$ & $0.0 \pm 1.9$ & - & - \\
\hline \multicolumn{5}{|c|}{ A 2052} \\
\hline$\# 0$ & $0.58 \pm 0.04$ & $0.7 \pm 0.4$ & $1.3 \pm 0.3$ & $0.8 \pm 0.3$ \\
\hline$\# 1$ & $0.41 \pm 0.04$ & $0.6 \pm 0.5$ & $1.4 \pm 0.5$ & $0.6 \pm 0.5$ \\
\hline \#2 & $0.13 \pm 0.06$ & - & $0.3 \pm 1.1$ & $3.3 \pm 2.6$ \\
\hline \multicolumn{5}{|c|}{ A 4059} \\
\hline$\# 0$ & $0.72 \pm 0.12$ & $0.5 \pm 0.6$ & $1.3 \pm 0.6$ & $1.7 \pm 0.8$ \\
\hline$\# 1$ & $0.44 \pm 0.08$ & $0.7 \pm 0.7$ & $1.4 \pm 0.9$ & $0.7 \pm 0.8$ \\
\hline$\# 2$ & $0.41 \pm 0.23$ & $1.2 \pm 2.5$ & $3.2 \pm 2.2$ & $1.7 \pm 2.5$ \\
\hline \multicolumn{5}{|c|}{ Hyd A } \\
\hline$\# 0$ & $0.42 \pm 0.05$ & $0.8 \pm 0.6$ & $1.1 \pm 0.5$ & $0.4 \pm 0.5$ \\
\hline$\# 1$ & $0.30 \pm 0.05$ & $0.9 \pm 0.8$ & $1.9 \pm 0.9$ & $0.7 \pm 0.8$ \\
\hline$\# 2$ & $0.20 \pm 0.11$ & - & $0.5 \pm 1.7$ & $0.0 \pm 1.2$ \\
\hline
\end{tabular}

a Integration region (\#0, \#1, \#2 for radii between 0-50, 50-200, and 200-500 in $h^{-1} \mathrm{kpc}$ ).

between the different exposures is small compared to the statistical errors: the r.m.s. deviations over the full spectral range are less than $30 \%$. Based on this, a $30 \%$ systematic error to the background counts was assigned.

The first order spectra from the RGS1 and RGS2 were fitted with the same model simultaneously. We limited the wavelength band to $8-28 \AA$, where the estimated background is typically less than $10 \%$ of the source counts. Therefore, the systematic uncertainty in the background modeling should not affect the results significantly.

In principle, the extracted spectrum contains photons from a range of different sky positions. However, in the cases of our sample, where the brightness is centrally peaked, the observed spectrum is dominated by the photons from the cluster 
Table 5. Abundances obtained from the EPIC analysis for each cluster (continued). Same notation as Table 4

\begin{tabular}{|c|c|c|c|c|}
\hline $\mathrm{R}$ & $\mathrm{Fe} / \mathrm{H}$ & $\mathrm{O} / \mathrm{Fe}$ & $\mathrm{Si} / \mathrm{Fe}$ & $\mathrm{S} / \mathrm{Fe}$ \\
\hline \multicolumn{5}{|c|}{ A 496} \\
\hline$\# 0$ & $0.60 \pm 0.05$ & $0.4 \pm 0.3$ & $1.6 \pm 0.3$ & $1.1 \pm 0.4$ \\
\hline$\# 1$ & $0.37 \pm 0.07$ & $1.5 \pm 1.2$ & $1.5 \pm 0.8$ & $0.7 \pm 0.8$ \\
\hline$\# 2$ & $0.41 \pm 0.26$ & - & $1.7 \pm 2.3$ & $1.8 \pm 2.7$ \\
\hline \multicolumn{5}{|c|}{ A 3112} \\
\hline$\# 0$ & $0.70 \pm 0.05$ & $0.9 \pm 0.6$ & $1.4 \pm 0.4$ & $0.4 \pm 0.3$ \\
\hline$\# 1$ & $0.36 \pm 0.05$ & $0.2 \pm 0.7$ & $1.0 \pm 0.8$ & $0.6 \pm 0.7$ \\
\hline$\# 2$ & $0.22 \pm 0.13$ & $0.0 \pm 1.6$ & $1.3 \pm 2.3$ & $2.3 \pm 2.7$ \\
\hline \multicolumn{5}{|c|}{ A 1795} \\
\hline$\# 0$ & $0.51 \pm 0.04$ & $0.7 \pm 0.4$ & $1.4 \pm 0.4$ & $0.6 \pm 0.4$ \\
\hline$\# 1$ & $0.32 \pm 0.04$ & $0.5 \pm 0.7$ & $1.9 \pm 1.1$ & $0.0 \pm 0.5$ \\
\hline$\# 2$ & $0.26 \pm 0.07$ & $1.4 \pm 1.9$ & $3.2 \pm 2.1$ & $0.6 \pm 1.2$ \\
\hline \multicolumn{5}{|c|}{ A 399} \\
\hline$\# 0$ & $0.63 \pm 0.35$ & $0.0 \pm 2.2$ & $0.0 \pm 1.2$ & $0.0 \pm 1.4$ \\
\hline$\# 1$ & $0.31 \pm 0.12$ & - & $0.9 \pm 2.2$ & - \\
\hline$\# 2$ & $0.28 \pm 0.13$ & - & - & - \\
\hline \multicolumn{5}{|c|}{ A 3266} \\
\hline$\# 0$ & $0.46 \pm 0.34$ & - & - & - \\
\hline \#1 & $0.27 \pm 0.13$ & - & - & - \\
\hline$\# 2$ & $0.22 \pm 0.08$ & - & - & - \\
\hline \multicolumn{5}{|c|}{ Perseus } \\
\hline$\# 0$ & $0.57 \pm 0.03$ & $1.4 \pm 0.3$ & $1.2 \pm 0.2$ & $0.4 \pm 0.4$ \\
\hline$\# 1$ & $0.41 \pm 0.04$ & $2.3 \pm 0.9$ & $1.2 \pm 0.4$ & $0.2 \pm 0.3$ \\
\hline \multicolumn{5}{|c|}{ Coma } \\
\hline$\# 0$ & $0.53 \pm 0.54$ & - & - & - \\
\hline$\# 1$ & $0.26 \pm 0.09$ & - & $3.8 \pm 2.8$ & $0.7 \pm 2.0$ \\
\hline$\# 2$ & $0.25 \pm 0.10$ & $2.4 \pm 2.8$ & $1.4 \pm 2.1$ & $0.0 \pm 0.9$ \\
\hline \multicolumn{5}{|c|}{ A 754} \\
\hline$\# 0$ & $0.32 \pm 0.34$ & $0.0 \pm 2.5$ & - & - \\
\hline$\# 1$ & $0.33 \pm 0.11$ & $1.1 \pm 2.2$ & $0.4 \pm 1.4$ & $0.6 \pm 1.6$ \\
\hline$\# 2$ & $0.21 \pm 0.09$ & - & - & - \\
\hline \multicolumn{5}{|c|}{ A 1835} \\
\hline$\# 0$ & $0.42 \pm 0.06$ & $2.0 \pm 1.4$ & $1.1 \pm 0.9$ & $0.6 \pm 1.0$ \\
\hline$\# 1$ & $0.38 \pm 0.09$ & $0.0 \pm 1.0$ & $0.0 \pm 0.6$ & $0.0 \pm 1.1$ \\
\hline$\# 2$ & $0.29 \pm 0.11$ & - & - & $1.7 \pm 2.8$ \\
\hline
\end{tabular}

core. By using a full instrument simulation, SciSim (the official $X M M$-Newton science simulation tool ${ }^{2}$ ) and the observed MOS image, we confirmed that the contamination from the cluster outer region (a projected radius from the cluster center larger than $3^{\prime}$ ) is less than $10 \%$ for a typical cluster and $20 \%$ at most for all clusters. This kind of contamination adds counts in the form of a continuum spectrum since lines are smeared out due to the large spatial extent of the outer cluster regions. This could make the obtained equivalent line width and hence the metal abundance relative to $\mathrm{H}$ appeared lower than the actual value by less than $10-20 \%$. The possible change of abundances relative to $\mathrm{Fe}($ e.g. $\mathrm{O} / \mathrm{Fe}$ ) should be much smaller. Since the thermal structure is mainly determined by the Fe-L line ratios, the effect of the contamination on the thermal structure should also be small. Therefore, we ignored this flux mixing and assumed that the values obtained from our spectral fits are emission-weighted values for the cluster core.

\footnotetext{
${ }^{2}$ see http://xmm.vilspa.esa.es
}

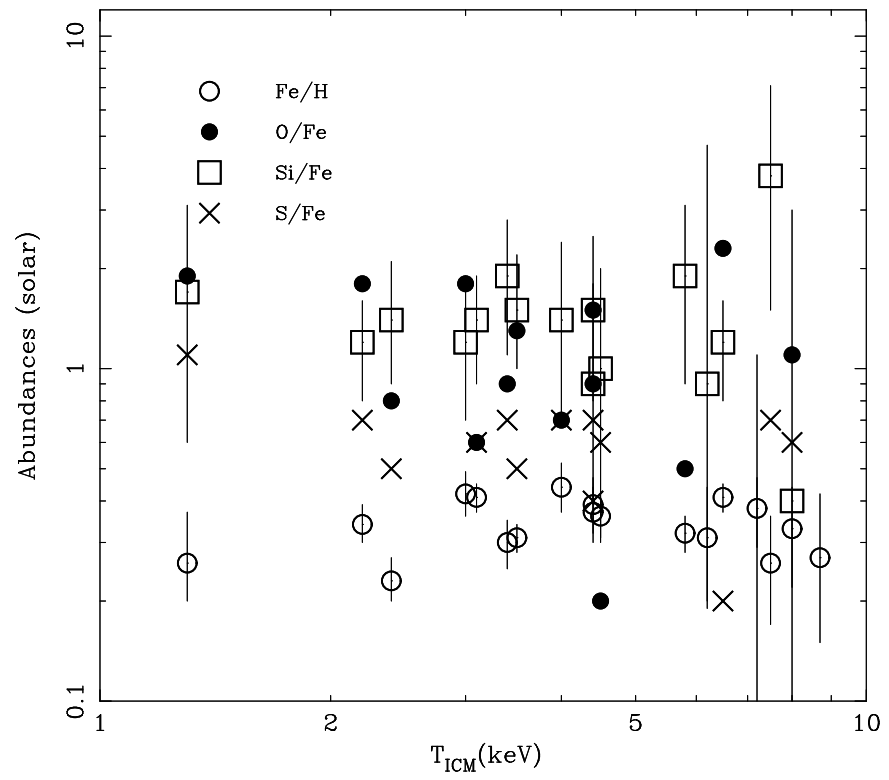

Fig. 6. Abundance vs temperature for each cluster averaged over (50-200) $h^{-1} \mathrm{kpc}$. Errors on O/Fe and S/Fe are not shown for clarity (These are presented in Tables 445). The data for MKW 9 are not shown for clarity, because this cluster has larger errors than the other clusters.

\subsection{Single-temperature model fitting}

We fitted a set of spectra for each cluster with the single temperature (1T) model. With this case, the free parameters are the normalization, $k T$, the $\mathrm{Fe}$ abundance, and the $\mathrm{Fe} / \mathrm{O}$ ratio. An exception is NGC 533, where the RGS spectrum is dominated by line emission. In this case, it is relatively difficult to determine the continuum level and hence the line equivalent widths. Therefore for NGC 533 we fixed the Fe abundance to the value obtained from the EPIC analysis above.

We obtained acceptable or almost acceptable fits $\left(\chi^{2} / \nu<\right.$ 1.2) with the $1 \mathrm{~T}$ model for Sérsic 159-3, A 4059, Hyd-A, A 496, A 3112, A 1795 and A 1835. In contrast, NGC 533 $\left(\chi^{2} / \nu=1.55\right)$, A $262(1.49)$, A 2052 (1.32), and 2A 0335 (1.48) exhibit clear deviations from the 1T model.

\subsection{Multi-temperature model fitting}

In some clusters, there is evidence for multi-temperature emission (Peterson et al. 2003). If there is a significant contribution from the cooler temperature components in the line emission, the abundances derived from the 1T model may differ from the true abundances.

To avoid this error, we used a multi-temperature model which consists of at most five temperature CIE components with fixed temperature separation, $\left(T_{0}=2 T_{1}=4 T_{2}=8 T_{3}=\right.$ $\left.16 T_{4}\right)$, but free normalization $\left(E M_{i}\right)$ for each component (the 5 T model); a similar approach was also taken by Peterson et al. (2003). In this case, the $\mathrm{Ne} / \mathrm{Fe}$ and $\mathrm{Mg} / \mathrm{Fe}$ ratios are also allowed to be free along with the $\mathrm{Fe} / \mathrm{H}$ and $\mathrm{O} / \mathrm{Fe}$ ratios. All temperature components were assumed to have the same abundances. 
Table 7. RGS fit results for a multi-temperature model. The mean abundances for the sample are also shown in the last row.

\begin{tabular}{lcccccc}
\hline target & $k T_{0}^{\mathrm{a}}$ & $\mathrm{O} / \mathrm{Fe}$ & $\mathrm{Ne} / \mathrm{Fe}$ & $\mathrm{Mg} / \mathrm{Fe}$ & $\mathrm{Fe} / \mathrm{H}$ & $\chi^{2} / \nu$ \\
\hline NGC 533 & $1.4 \pm 0.5$ & $0.54 \pm 0.15$ & $1.3 \pm 0.7$ & $1.7 \pm 1.0$ & 0.50 & $264 / 226$ \\
A 262 & $2.4 \pm 0.7$ & $0.64 \pm 0.12$ & $0.6 \pm 0.5$ & $0.1 \pm 0.5$ & $0.58 \pm 0.11$ & $229 / 231$ \\
Sérsic 159-3 & $2.3 \pm 0.2$ & $0.67 \pm 0.15$ & $0.8 \pm 0.4$ & $0.6 \pm 0.5$ & $0.38 \pm 0.03$ & $279 / 263$ \\
A 2052 & $2.8 \pm 0.3$ & $0.66 \pm 0.10$ & $0.8 \pm 0.3$ & $0.9 \pm 0.6$ & $0.68 \pm 0.08$ & $284 / 263$ \\
2A 0335 & $1.6 \pm 0.1$ & $1.00 \pm 0.23$ & $0.4 \pm 0.5$ & $1.0 \pm 0.6$ & $0.29 \pm 0.04$ & $282 / 188$ \\
A 4059 & $3.5 \pm 0.7$ & $0.68 \pm 0.16$ & $1.4 \pm 0.7$ & $0.6 \pm 0.8$ & $0.58 \pm 0.10$ & $215 / 181$ \\
Hydra A & $2.9 \pm 0.3$ & $0.70 \pm 0.18$ & $0.5 \pm 0.6$ & $0.0 \pm 0.3$ & $0.37 \pm 0.06$ & $230 / 228$ \\
A 496 & $3.2 \pm 0.6$ & $0.66 \pm 0.15$ & $1.2 \pm 0.6$ & $1.1 \pm 0.9$ & $0.55 \pm 0.14$ & $236 / 229$ \\
A 3112 & $4.2 \pm 0.8$ & $0.63 \pm 0.18$ & $0.5 \pm 0.6$ & $0.0 \pm 0.4$ & $0.69 \pm 0.14$ & $189 / 225$ \\
A 1795 & $6.4 \pm 1.0$ & $0.78 \pm 0.14$ & $1.5 \pm 0.4$ & $1.4 \pm 0.8$ & $0.60 \pm 0.13$ & $284 / 261$ \\
A 1835 & $4.6 \pm 1.0$ & $0.35 \pm 0.24$ & $0.9 \pm 0.6$ & $0.0 \pm 0.7$ & $0.49 \pm 0.12$ & $295 / 284$ \\
\hline mean & - & $0.63 \pm 0.05$ & $1.0 \pm 0.2$ & $0.66 \pm 0.2$ & $0.54 \pm 0.03$ & - \\
\hline
\end{tabular}

a Temperature of the hottest component in $\mathrm{keV}$.

The results are shown in Table7 7 In Sérsic 159-3, A 4059, Hyd-A, A 3112 and A 1835 where the 1T model already provided an acceptable fit, this model does not improve the fit. The derived abundances are consistent with those of the $1 \mathrm{~T}$ fit, simply because the same temperature component dominates the emission in both the 1T and 5T models.

In 2A 0335+096, we could not obtain an acceptable fit even with the 5T model. We note that the EPIC spectra of the inner region of this cluster also show a deviation from the $2 \mathrm{~T}$ model (Table 3). The Galactic absorption towards this cluster is by far larger than that of other clusters. Furthermore, the X-ray image shows a complex structure (Sarazin et al.1992). Therefore, we assumed that our spectral and spatial modeling is oversimplified for this cluster. Note that we exclude the EPIC results for the inner region of this cluster in our discussion.

In other clusters, the $5 \mathrm{~T}$ model improves the fit significantly. The best example is A 262, where the model reproduced the observed spectra very well. By using this model, we obtained acceptable fits $\left(\chi^{2} / \nu<1.2\right)$ for all clusters. While there are significant changes in the derived $\mathrm{Fe}$ abundance in some cases, the change in the $\mathrm{O} / \mathrm{Fe}$ ratio is at most $30 \%$ or 0.2 times solar, compared to the $1 \mathrm{~T}$ fitting.

Peterson et al. (2003) analyzed a similar RGS data set, focusing on the temperature structure. Our model for the temperature structure is basically consistent with their results.

In Fig. 2. we compare the $\mathrm{O}$ and $\mathrm{Fe}$ abundance as obtained by EPIC and RGS. In 2A 0335+096, the $\mathrm{Fe} / \mathrm{H}$ ratio derived from the RGS data is two times smaller than that derived from the EPIC data, while the $\mathrm{O} / \mathrm{Fe}$ ratio from the two instruments is consistent. We presume that this discrepancy is due to the nature of the central spectra which is more complex than our modeling. Although the errors in the oxygen abundance determination from EPIC is large, apart from 2A 0335+096, the two instruments provide almost consistent results: a formal fit of the $\mathrm{Fe}$ abundance from RGS on the Fe abundance from EPIC to the $1: 1$ relation yields $\chi^{2} / \nu=7.68 / 10$.

\section{Summary and Discussion}

\subsection{Present results}

Using spatially-resolved EPIC spectra, we have resolved the temperature and abundance distribution on a sub-arcmin scale in a sample of 19 clusters. Furthermore, using high spectral resolution RGS spectra of 11 cluster cores, we have resolved the soft X-ray spectrum dominated by the Fe-L and the O VIII Ly $\alpha$ lines. This provides not only tight constrains on the thermal structure but also a robust measurement of the $\mathrm{O}$ abundance. Below we summarize our main results.

1. We found no significant variation in the $\mathrm{Fe}$ abundance and the $\mathrm{Si}, \mathrm{S}$, and $\mathrm{O}$ ratios to the $\mathrm{Fe}$ among the systems (see Fig. 6.

2. The $\mathrm{O} / \mathrm{H}$ and $\mathrm{O} / \mathrm{Fe}$ ratio in the cluster cores, (2060) $h^{-1} \mathrm{kpc}$ in radius, are $0.34 \pm 0.03$ and $0.63 \pm 0.05$, respectively. The r.m.s. deviation for individual clusters as compared to these average values is comparable to the measurement errors on these ratios.

3. In all clusters with a temperature less than $6 \mathrm{keV}$, except for the faint cluster MKW 9, we detected a central increase in the Fe abundance; the abundance starts to increase at (50100) $h^{-1} \mathrm{kpc}$ in radius from $0.2-0.4$ solar value to $0.6-$ 0.8 solar value towards the center.

4. The $\mathrm{Si} / \mathrm{Fe}$ and $\mathrm{S} / \mathrm{Fe}$ ratios in cool and medium temperature clusters are radially uniform within $(200-500) h^{-1} \mathrm{kpc}$ with mean values of $1.4 \pm 0.2$ and $1.1 \pm 0.3$, respectively, relative to the solar ratio.

5. Contrary to the $\mathrm{Fe}, \mathrm{Si}$, and $\mathrm{S}$ abundances, the $\mathrm{O}$ abundance shows no spatial variations. The errors on the $\mathrm{O}$ abundance for each cluster are large. However, when we combine the results from several clusters and assume no correlation of the systematic errors of the measurements between clusters, we detected a significant radial variation in the $\mathrm{O} / \mathrm{Fe}$ ratio; the mean $\mathrm{O} / \mathrm{Fe}$ ratios are $0.5_{-0.1}^{+0.3}, 1.7_{-0.4}^{+0.9}$, and $4.1_{-1.9}^{+3.5}$ for $(0-50) h^{-1} \mathrm{kpc},(50-200) h^{-1} \mathrm{kpc}$, and (200-500) $h^{-1} \mathrm{kpc}$, respectively. 


\subsection{Similarity of the abundance among clusters}

ASCA and BeppoSAX observations have established the Fe increase towards the cluster center in relatively cool clusters with cD galaxies (e.g. Fukazawa et al.2000, De Grandi and Molendi 2001). Our measurement not only confirms these results in the cool and medium temperature clusters but also suggests a similar enhancement in hot clusters without a prominent $\mathrm{cD}$ galaxy (e.g. Coma), probably because of the improved spatial resolution.

Beside the Fe abundance, we found no variation in the $\mathrm{Si}$ and other element ratios to $\mathrm{Fe}$ among the clusters. This implies a homogeneous production of the metals in our sample. However, the sample is biased towards medium-temperature $\mathrm{X}$-ray luminous clusters. Therefore we make no conclusion about the universal distribution of the relative abundances. In fact Fukazawa et al. (1998) claimed an increase in Si/Fe from cool to hot systems using 40 clusters.

\subsection{The $O$ abundance at the cluster core}

The theoretical models for supernova (SN) types Ia and II predict the $\mathrm{O} / \mathrm{Fe}$ ratio to be $<0.05$ and $1.5-4$ times solar values, respectively. On the other hand, the observed $\mathrm{O} / \mathrm{Fe}$ ratio at the cluster cores are between these two predicted values. This suggests that the ICM metals at the cluster cores could be produced by a mix of both types of SNe. A value of the O/Fe abundance ratio in the ICM close to solar values supports the idea that the metals in the ICM and the Galaxy were produced in a similar way. Assuming the $\mathrm{O}$ and Fe yields of SN Ia and II from Tsujimoto et al. (1995), the observed O/Fe ratio $(\sim 0.65)$ can be reproduced by the relative frequencies of the two $\mathrm{SNe}$, $N_{\text {Ia }} / N_{\text {II }}$, of $\sim 0.6$. In this model, the mass fractions of the $\mathrm{O}$ and Fe originated from SN Ia are 0.05 and $\sim 0.8$, respectively.

The observed $\mathrm{O}$ abundance provides an accurate way to determine the total mass of $\mathrm{O}$ in the ICM, $M_{\mathrm{O}}^{\mathrm{ICM}}$. We estimate $M_{\mathrm{O}}^{\mathrm{ICM}}$ within $50 h^{-1} \mathrm{kpc}$ to range from $10^{8}$ (NGC 533) to $2 \times 10^{9}$ (A 1795) in units of $h^{-2.5} \mathrm{M}_{\odot}$ for our RGS sample. We assume that all oxygen was produced in SNe II. Then, combining $M_{\mathrm{O}}^{\mathrm{ICM}}$ with a SN II model O yield $\left(\sim 2 \mathrm{M}_{\odot}\right.$, Tsujimoto et al.1995), gives a total number of SNe II of $10^{8}-2.5 \times 10^{9}$ for $h=0.7$. Further assuming that the duration of SNe II activity is $10^{7} \mathrm{yr}$ ( a typical life time of a $20 \mathrm{M}_{\odot}$ star), we can estimate the SN II rate to be $10-200 \mathrm{SN}$ IIe $\mathrm{yr}^{-1}$. This rate is at least one or two orders of magnitude larger than that of a typical starburst galaxy. In other words, the observed $\mathrm{O}$ mass requires a large amount of massive star formation, producing the corresponding number of SNe II.

The observed $\mathrm{O} / \mathrm{H}$ ratio in cluster cores $(20-60 \mathrm{kpc}$ in radius) is about half that observed in the central regions (5$15 \mathrm{kpc}$ ) of giant elliptical galaxies, like in M87 (Sakelliou et al. 2002, Gastaldello and Molendi 2002, Finoguenov et al. 2002) and NGC 4636 (Xu et al. 2002). The overabundance of oxygen in galaxy centers suggests that in those regions oxygen has been produced via massive star formation and that metals have not been completely mixed with the ambient gas (Gastaldello and Molendi 2002).

\subsection{The total Fe mass in clusters}

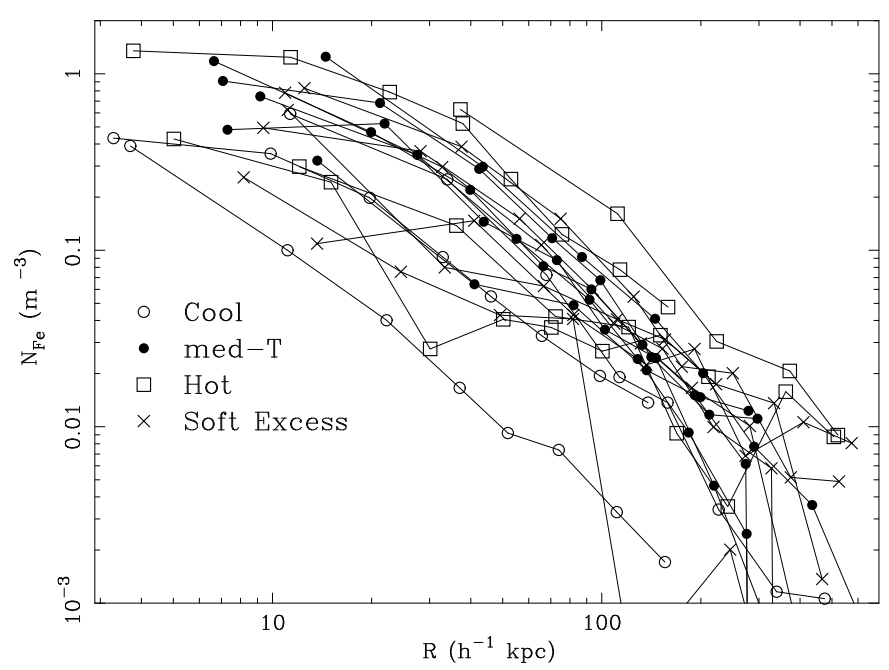

Fig. 7. The Fe number density in the ICM. Same notation as Fig. 5.

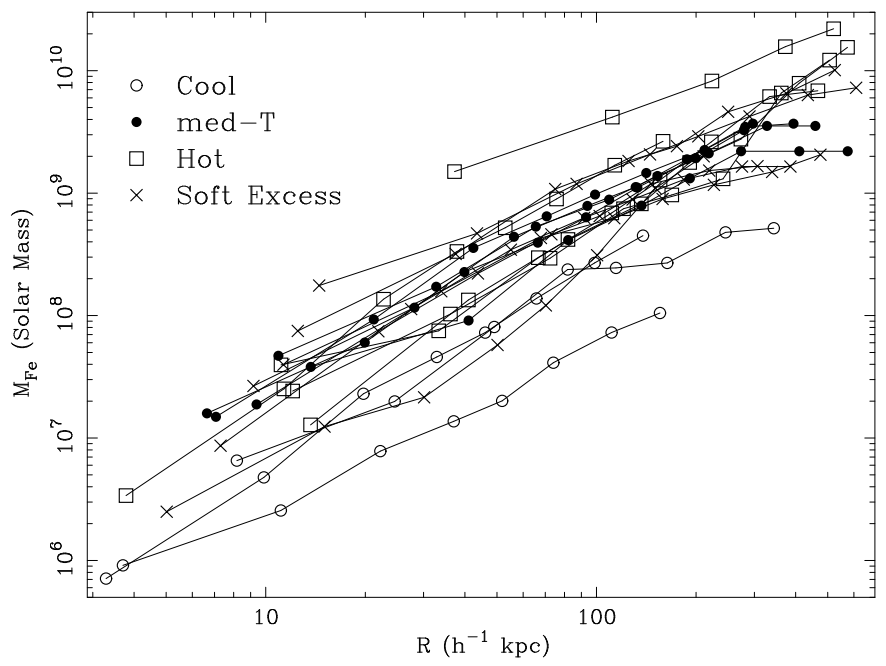

Fig. 8. Same as the previous figure, but for the integrated Fe mass in the ICM.

Table 8. The iron mass to light ratio within $250 h^{-1} \mathrm{kpc}$.

\begin{tabular}{lccc}
\hline cluster & $\begin{array}{c}M_{\mathrm{Fe}} \\
\left(10^{9} \mathrm{M}_{\odot}\right)\end{array}$ & $N_{0.5}^{\mathrm{a}}$ & $\begin{array}{c}M_{\mathrm{Fe}} / L_{\mathrm{B}} \\
\left(10^{-2} \mathrm{M}_{\odot} / \mathrm{L}_{\odot}\right)\end{array}$ \\
\hline MKW 9 & 0.5 & 13 & $0.3 \pm 0.15$ \\
MKW 3s & 1.5 & 13 & $0.9 \pm 0.2$ \\
A 496 & 2.8 & 14 & $1.5 \pm 0.5$ \\
A 1795 & 6.0 & 27 & $1.0 \pm 0.2$ \\
A 754 & 3.8 & 29 & $0.7 \pm 0.3$ \\
\hline
\end{tabular}

a Number of galaxies within a radius of $250 h^{-1} \mathrm{kpc}$. 
We also calculated the total Fe density and mass in the ICM as a function of radius, based on our measurements of the emission measure and the $\mathrm{Fe}$ abundances (Figs. 7 and 8 ). To compare this $\mathrm{Fe}$ mass and the predicted $\mathrm{Fe}$ production rate in galaxies, we estimate the iron mass to light ratio (IMLR). We use the galaxy number within a radius of $250 h^{-1} \mathrm{kpc}$, $N_{0.5}$ (Bahcall 1981 and reference therein), and estimate the total optical luminosity of member galaxies based on the relation $L_{B}=4.5 \times 10^{9} N_{0.5}^{1.42} h^{-2} \mathrm{~L}_{\odot}$ (Edge and Stewart 1991). Table 8 shows the results for representative clusters, where $N_{0.5}$ is available.

The IMLR of MKW 9 is relatively smaller than for other clusters. The potential of MKW 9 might not be as deep as in other systems and a large part of the metal-enriched gas has been driven out already from the potential well. An IMLR of order $10^{-2}$ in other systems is consistent with previous measurements (e.g. Tsuru 1991) and at least a few times larger than the predicted value based upon the current rate of SN Ia in elliptical galaxies, as argued previously (e.g., Tsuru 1991, Renzini et al. 1993). We presume that a large fraction of Fe has been produced also in the early phase of the galaxy formation with a high production rate. Relatively low metal abundances observed in elliptical galaxies (sub-solar at most; e.g. Matsushita et al. 2000) also support this idea.

\subsection{Spatial distribution of the $\mathrm{O}, \mathrm{Si}, \mathrm{S}$, and $\mathrm{Fe}$ abundances}

The most simple model to explain the results (3) and (4) is that $\mathrm{Si}, \mathrm{S}$, and $\mathrm{Fe}$ have a common origin for a large part, while $\mathrm{O}$ has a different origin. This contrast between $\mathrm{Si}-\mathrm{S}-\mathrm{Fe}$ and $\mathrm{O}$ has reported separately in A 496 (Tamura et al. 2001b). Qualitatively this trend is consistent with the current idea that the contribution from SN Ia relative to that from SN II, $N_{\mathrm{Ia}} / N_{\mathrm{II}}$, increases towards the cluster center and the $\mathrm{O} / \mathrm{Fe}$ ratio is more sensitive to $N_{\mathrm{Ia}} / N_{\mathrm{II}}$ than the $\mathrm{Si} / \mathrm{Fe}$ ratio. We do not attempt a further detailed comparison between the observation and models, because of large uncertainties in both the data (e.g. the $\mathrm{O} / \mathrm{Fe}$ ratio in the outer regions and for hot clusters) and the theory (e.g. Gibson et al. 1997).

Acknowledgements. This work is based on observations obtained with $X M M-N e w t o n$, an ESA science mission with instruments and contributions directly funded by ESA Member States and the USA (NASA). The Space Research Organisation of the Netherlands (SRON) is supported financially by NWO, the Netherlands Organisation for Scientific Research. We thank the referee for useful comments.

\section{References}

Anders, E., Grevesse, N., 1989, Geochim. Cosmochim. Acta 53, 197 Arnaud, M., Rothenflug, R., Boulade, O., Vigroux, L., \& VangioniFlam, E. 1992, A\&A, 254, 49

Bahcall, N. A. 1981, ApJ, 247, 787

Buote, D.A., 2000, MNRAS, 311, 176

David, L. P., Nulsen, P. E. J., McNamara, B. R., Forman, W., Jones, C., Ponman, T., Robertson, B., \& Wise, M. 2001, ApJ, 557, 546

Dickey, J.M., \& Lockman, F.J., 1990, ARA\&A 28, 215

Edge, A.C., \& Stewart, G.C. 1991, MNRAS, 252, 428
Finoguenov, A., David, L.P., Ponman, T.J., 2000, ApJ, 544, 188

Finoguenov, A., Matsushita, K., Böhringer, H., Ikebe, Y., \& Arnaud, M. 2002, A\&A, 381, 21

Fukazawa, Y., Makishima, K., Tamura, T., et al. 1998, PASJ, 50, 187

Fukazawa, Y., Makishima, K., Tamura, T., Nakazawa, K., Ezawa, H., Ikebe, Y., Kikuchi, K., \& Ohashi, T. 2000, MNRAS, 313, 21

Gasteldello, F. \& Molendi, S. 2002, ApJ, 572, 160

Gibson, B. K., Loewenstein, M., \& Mushotzky, R. F. 1997, MNRAS, 290,623

De Grandi, S. and Molendi, S., 2001, ApJ, 551, 153

den Herder, J.W., Brinkman, A.C., Kahn, S.M., et al. 2001, A\&A, 365, L7

Jansen, F., Lumb, D., Altieri, B., et al. 2001, A\&A, 365, L1

Kaastra, J.S. 1992, An X-Ray Spectral Code for Optically Thin Plasmas (Internal SRON-Leiden Report, updated version 2.0)

Kaastra, J.S., Ferrigno, C., Tamura, T., et al. 2001, A\&A, 365, L99

Kaastra, J.S., Lieu, R., Tamura, T., Paerels, F.B.S., den Herder, J.W. 2003a, A\&A, 397, 445

Kaastra, J.S., Tamura, T., Peterson, J.R., et al., 2004, A\&A, 413, 415

Kaastra, J.S., Lieu, R., Tamura, T., Paerels, F.B.S., den Herder, J.W. 2003c, Adv. Sp. Res., in press.

Liedahl, D.A., Osterheld, A.L., \& Goldstein, W.H. 1995, ApJL, 438, 115

Matsushita, K., Ohashi, T., \& Makishima, K. 2000, PASJ, 52, 685

Matsushita, K., Finoguenov, A., \& Böhringer, H. 2003, A\&A, 401, 443

Mewe, R., Gronenschild, E.H.B.M., and van den Oord, G.H.J. 1985, A\&AS, 62, 197

Molendi, S., \& Gastaldello, F., 2001, A\&A, 375, L14

Nevalainen, J., Lieu, R., Bonamente, M., \& Lumb, D., 2003, ApJ, 584, 716

Peterson, J.R., Paerels, F.B.S., Kaastra, J.S., et al., 2001, A\&A, 365, L104

Peterson, J.R., Kahn, S.M., Paerels, F.B.S., et al. 2003, ApJ, 590, 207

Renzini, A., Ciotti, L., D’Ercole, A. \& Pellegrini, S., ApJ, 1993, 419

Sarazin, C. L., O'Connell, R. W., \& McNamara, B. R. 1992, ApJL, 397, L31

Sakelliou, I., Peterson, J.R., Tamura, T., et al. 2002, A\&A, 391, 903

Strüder, L., Briel, U., Dennerl, K., et al. 2001, A\&A, 365, L18

Tamura, T., Kaastra, J.S., Peterson, J.R., et al., 2001a, A\&A, 365, L93

Tamura, T., Bleeker, J.A.M., Kaastra, J.S., et al. 2001b, A\&A 379, 107

Tamura, T., den Herder, J.W., \& González-Riestra, R., 2003, XMM calibration note, XMM-SOC-CAL-TN-0034 (http://xmm.vilspa.esa.es/)

Tsujimoto, T., Nomoto, K., Yoshii, Y., Hashimoto, M., Yanagida, S., \& Thielemann, F.-K. 1995, MNRAS, 277, 945

Tsuru, T. 1991, PhD thesis, University of Tokyo

Turner, M.J., Abbey, A., Arnaud, M., et al. 2001, A\&A, 365, L27

$\mathrm{Xu}$, H. et al. 2002, ApJ, 579, 600 

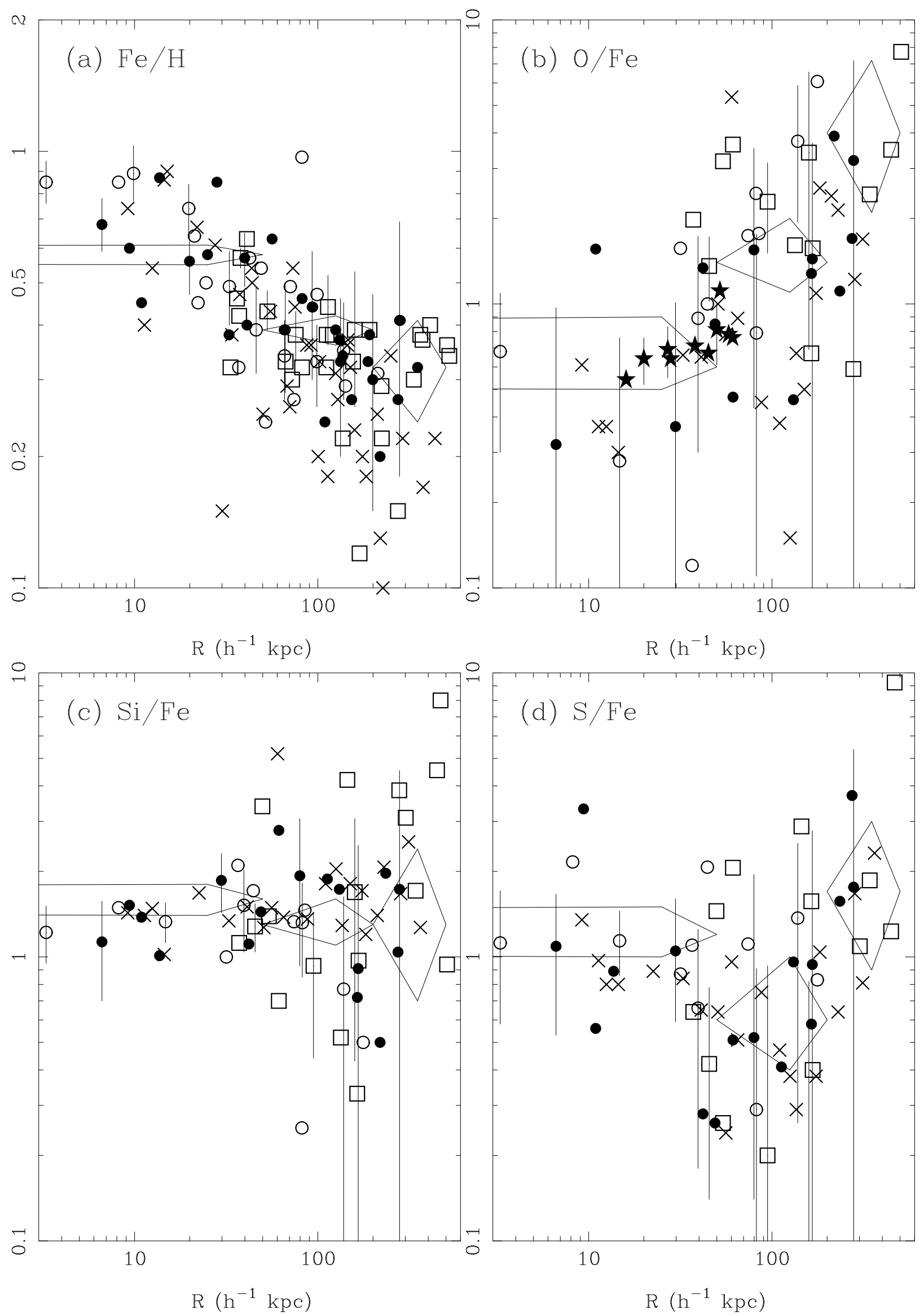

Fig. 5. Radial profiles of the $\mathrm{Fe} / \mathrm{H}, \mathrm{O} / \mathrm{Fe}, \mathrm{Si} / \mathrm{Fe}$ and $\mathrm{S} / \mathrm{Fe}$ ratios in solar units, derived from the EPIC spectra. The best-fit values for cool, medium-temperature, hot, and soft excess clusters are shown with open-circles, filled-circles, open-square, and " $\times$ ", respectively. Typical error bars are shown for A 262, A 496, and Perseus. Mean values and errors for the cool and mediumtemperature clusters $(\mathrm{T}<6 \mathrm{keV})$, except for the soft excess clusters, are shown with diamonds. In panel (b), the O/Fe ratio obtained from the RGS spectra are also shown with filled-stars. 
arXiv:astro-ph/0403058 v1 2 Mar 2004

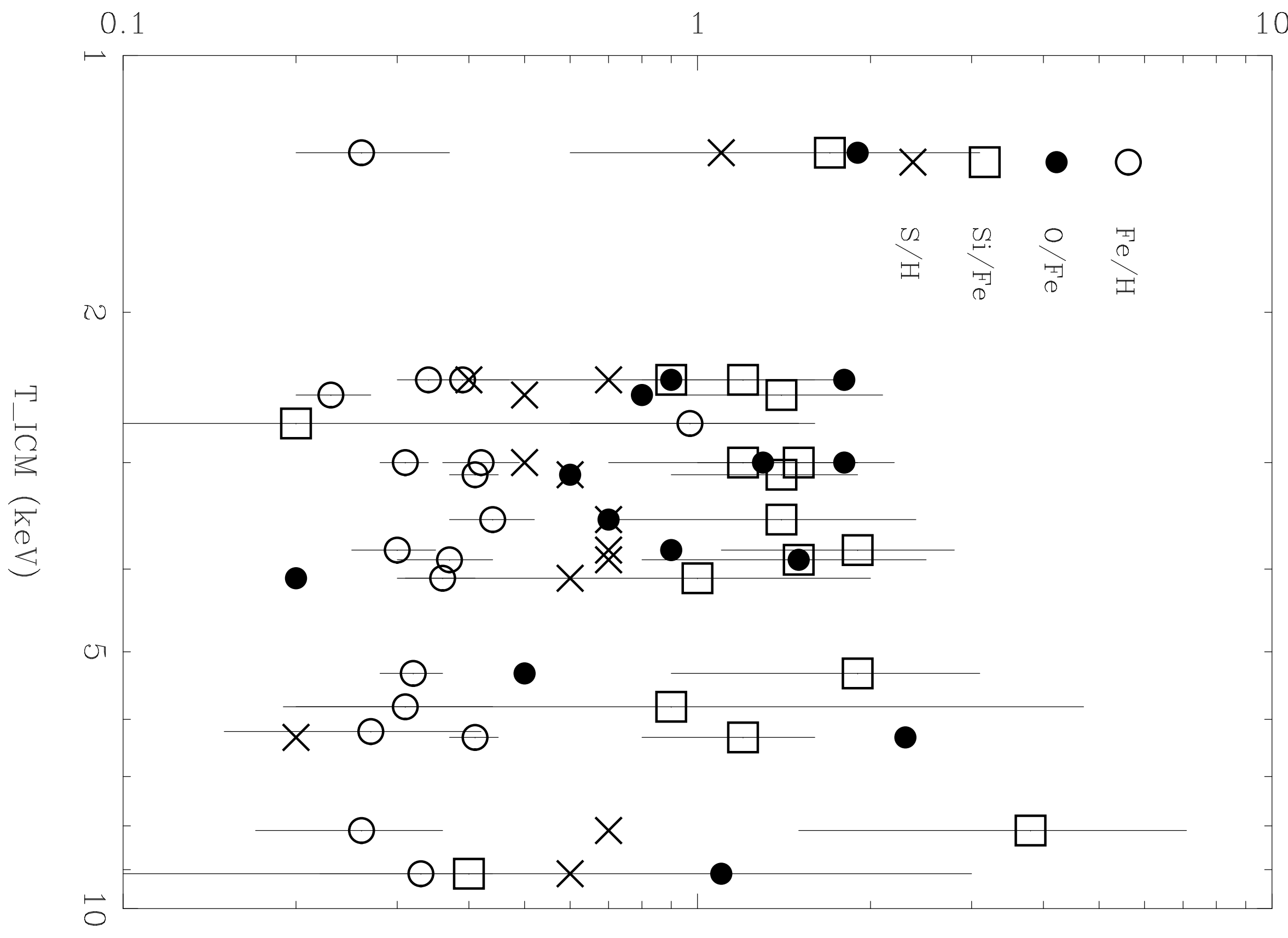

